\title{
Strain-Dependent Induction of Human Enterocyte Apoptosis by Blastocystis Disrupts Epithelial Barrier and ZO-1 Organization in a Caspase 3- and 9-Dependent Manner
}

\author{
Zhaona Wu, Haris Mirza, Joshua D. W. Teo, and Kevin S. W. Tan \\ Laboratory of Molecular and Cellular Parasitology, Department of Microbiology, Yong Loo Lin School of Medicine, \\ National University of Singapore, 5 Science, Drive 2, Singapore 117596
}

Correspondence should be addressed to Kevin S. W. Tan; kevin_tan@nuhs.edu.sg

Received 21 November 2013; Accepted 4 March 2014; Published 14 April 2014

Academic Editor: Marlene Benchimol

Copyright $\odot 2014$ Zhaona Wu et al. This is an open access article distributed under the Creative Commons Attribution License, which permits unrestricted use, distribution, and reproduction in any medium, provided the original work is properly cited.

\begin{abstract}
Blastocystis is an emerging protistan parasite colonizing the human intestine. It is frequently reported to cause general intestinal symptoms of vomiting, diarrhea, and abdominal pain. We recently demonstrated that Blastocystis rearranged cytoskeletal proteins and induced intestinal epithelial barrier compromise. The effect of Blastocystis on enterocyte apoptosis is unknown, and a possible link between microbially induced enterocyte apoptosis and increased epithelial permeability has yet to be determined. The aim of this study is to assess if Blastocystis induces human enterocyte apoptosis and whether this effect influences human intestinal epithelial barrier function. Monolayers of polarized human colonic epithelial cell-line Caco-2 were incubated with Blastocystis subtype 7 and subtype 4 . Assays for both early and late markers of apoptosis, phosphatidylserine externalization, and nuclear fragmentation, respectively, showed that Blastocystis ST-7, but not ST-4, significantly increased apoptosis in enterocytes, suggesting that Blastocystis exhibits host specificity and strain-to-strain variation in pathogenicity. ST-7 also activated Caco-2 caspases 3 and 9 but not 8. ST-7 induced changes in epithelial resistance, permeability, and tight junction (ZO-1) localization. Pretreatment of Caco2 monolayers with a pan-caspase inhibitor $\mathrm{z}$-VAD-fmk significantly inhibited these changes. This suggests a role for enterocyte apoptosis in Blastocystis-mediated epithelial barrier compromise in the human intestine.
\end{abstract}

\section{Introduction}

Blastocystis is an anaerobic protistan parasite of the gut [1$3]$. It is a species complex comprising of 17 subtypes out of which at least 9 are known to infect humans [4]. Other common hosts are rats, pigs, and chickens [1]. It is one of the most common parasites found in humans [3] with prevalence ranging from $10 \%$ in developed countries to $50 \%$ in developing countries [3]. Infection with the parasite is associated with common intestinal symptoms of mucous and watery diarrhea, bloating, abdominal pain, and vomiting [3]. It has a higher prevalence in impoverished children [5] and patients immunocompromised due to HIV infection [6] or malignancy [7], suggesting opportunistic pathobiology [3]. It is often associated with irritable bowel syndrome [8-11] and urticaria $[12,13]$. It is also considered one of the causes of traveler's diarrhea [14]. Chronic and recurrent infections are common despite prolonged antimicrobial treatment [13, 15]. Recent studies suggest that in some cases the parasite is also capable of epithelial invasion [16-19].

Despite being discovered more than 100 years ago [20, 21] as well as the recent advances in our understanding of this organism regarding its potential virulence factors and host responses $[15,22]$, its pathogenic potential remains controversial [4], owing to frequent reports of asymptomatic carriage, nonresponsiveness to chemotherapy, coinfections with other known pathogens, and high degree of genetic and pathobiological diversity within and across subtypes [23]. Strain-to-strain variation in virulence of the parasite has been reported in clinical and animal infection studies $[8,24$, 25]. We recently reported that a subtype-7 (ST-7) isolate of Blastocystis recovered from a symptomatic patient exhibits higher cysteine protease and arginase activity (potential parasite virulence factors) compared to ST-4 isolates $[15,26]$. 
ST-7 strain also exhibited higher immune evasion potential in inducing degradation of secretory IgAs and downregulation of epithelial antiparasitic nitric oxide production [15, 27]. However, direct evidence of comparative study in pathogenic effects of different Blastocystis strains on the host cells with a well-recognized host-pathogen interaction mode is still limited.

Modulation of intestinal epithelial barrier function is one of the major mechanisms employed by pathogens to induce host pathology [28]. Organisms ranging from viruses [29] to bacteria [30] as well as parasites such as Giardia [31] and Entamoeba [32] are known to compromise epithelial barrier function. Compromise of epithelium's barrier function exposes subepithelial tissue to toxic luminal contents [33], which could lead to dire consequences for the host. Interestingly, various luminal parasites sabotage this gate function by utilizing the apoptotic machinery of the host cell [34, 35]. Apoptosis or programmed cell death is a mechanism of clearing up unwanted cells by the body while simultaneously limiting overt immune response [36]. Entamoeba induces enterocyte apoptosis to facilitate the parasite infection of gut [37] while Giardia induces epithelial barrier compromise by activating caspase 3-mediated enterocyte programmed cell death [38]. There is ample evidence suggesting that Blastocystis causes intestinal epithelial barrier compromise in vitro and in vivo $[22,25,39]$. Rodent Blastocystis has been shown to induce caspase-mediated apoptosis, with no effect on the barrier function of rat intestinal epithelium [39]. An association of parasite-induced apoptosis with disruption of epithelial barrier function, as observed in Giardia and Entamoeba infections, is not known in human intestinal epithelium.

By using a human intestinal Caco- 2 cell line that has been well established as an in vitro model system to study Blastocystis-host interaction $[4,15,22]$, we report for the first time that Blastocystis subtype 7 (ST-7; isolate B), recovered from a symptomatic patient, induces breakdown of epithelial barrier function and caspase-3- and 9-mediated apoptosis and rearrangement of the tight junction associated protein $\mathrm{ZO}-1$. In contrast, a subtype recovered from a rat (ST-4; isolate WR-1), previously reported to induce rat epithelial injury [39], did not cause any pathology in human epithelium. In this study, caspase inhibition in human gut epithelium prevented the effect of parasite on barrier dysfunction. This contrasts with the previous study involving rat epithelial cells, in which caspase inhibition did not rescue cells from barrier compromise [39].

\section{Materials and Methods}

2.1. Culture of Caco-2 Colonic Epithelial Cell Line. All Blastocystis-host interaction experiments were performed using Caco-2 human colonic cell line (ATCC). Caco-2 stock cultures were maintained in T-75 flasks in a humidified incubator with $5 \% \mathrm{CO}_{2}$ at $37^{\circ} \mathrm{C}$. Cell cultures were grown in Dulbecco's modified Eagle's medium (HyClone) supplemented with $10 \%$ heat-inactivated fetal bovine serum (HyClone) and $1 \%$ each of sodium pyruvate, MEM, and antibiotic
"Penstrep" (Gibco). Culture health was evaluated using the Trypan blue assay and only cultures with $>95 \%$ viability were used for the experiments. Cells were trypsinized with $0.25 \%$ trypsin-EDTA. Cell cultures for western blotting experiments were grown on standard cell-culture 6-well plates (Corning). For confocal imaging, cells were cultured on poly-L-lysine treated $12 \mathrm{~mm}$ glass coverslips, placed in standard 6-well culture plates (Corning). Cell cultures for annexin-V-FITC apoptosis assay were grown on standard 24-well cell-culture plates (Corning). For transepithelial resistance (TER) and permeability experiments, cells were grown on Millipore transwell filters with PET membranes of $3 \mu \mathrm{m}$ pore size placed in 24-well tissue culture plates. In order to synchronize cells before experiments, all cultures were serum-starved overnight in antibiotic free and serum free DMEM. For caspase inhibition experiments, cell cultures were pretreated with $40 \mu \mathrm{M}$ broad-spectrum caspase inhibitor, z-VAD-fmk (Sigma) for $4 \mathrm{~h}$. Cytochalasin D (Sigma) was used as a positive control for epithelial resistance and permeability experiments at a concentration of $1 \mu \mathrm{g} / \mathrm{mL}$. Staurosporine was used as a positive control for apoptosis and $\mathrm{ZO}-1$ rearrangement experiments at a concentration of $0.5 \mu \mathrm{M}$.

2.2. Parasite Culture and Lysate. Two axenized Blastocystis isolates belonging to different subtypes (ST) were used in this study. Isolate B, belonging to ST-7, was isolated from a symptomatic patient at the Singapore General Hospital [26], while isolate WR-1, belonging to ST-4, was isolated from a Wistar rat during an animal survey [26]. Both ST-7 and ST4 represent zoonotic subtypes. They are frequently isolated from stool samples. ST-7 is often associated with intestinal symptoms and has been known to induce epithelial barrier disruption $[8,22]$. Other than humans, common hosts for ST7 and ST-4 isolates are birds and rats, respectively [40].

Parasite cultures were maintained in prereduced Iscove's modified Dulbecco's medium (IMDM) supplemented with $10 \%$ heat-inactivated horse serum. Cultures were kept under anaerobic conditions in an Anaerojar (Oxoid) with gas pack (Oxoid) at $37^{\circ} \mathrm{C}$. Only log-phase cultures were harvested for lysate preparation. The cultures were washed twice in sterile PBS. Parasitic lysates were prepared by three freezethaw cycles in liquid nitrogen and $37^{\circ} \mathrm{C}$ water bath. Unless otherwise indicated, monolayers in all experiments were incubated with $10^{8}$ parasite/mL lysate.

2.3. Epithelial Resistance. Transepithelial resistance (TER) across Caco-2 monolayer was measured using MilliporeERS-2 Volt-Ohm-Meter. Caco-2 monolayers were grown on Millipore transwell system as described above and TER was measured every alternate day until it peaked ( day 21 ; $\left.1000 \Omega / \mathrm{cm}^{2}\right)$. Blastocystis ST-7 and Blastocystis ST-4 were coincubated with epithelium for 3, 6, 12, and 24 h. For dosedependent experiments, monolayers were coincubated with 0.25 to $2 \times 10^{8}$ parasites $/ \mathrm{mL}$ for $24 \mathrm{~h}$. After incubation, monolayers were carefully washed twice with Hank's balanced salt solution (HBBS). $200 \mu \mathrm{L}$ of warm media was added to the apical compartment before taking TER measurements. 
All measurements were done at $37^{\circ} \mathrm{C}$ to minimize reading fluctuations.

2.4. Epithelial Permeability. Caco-2 monolayers were cultured on a transwell system as described earlier till they reach confluency and tight junction maturation on day 21. After confirmation of maturation by TER measurement, monolayers were coincubated with parasites for $24 \mathrm{~h}$. Following coincubation, epithelial and basolateral compartments were washed twice, followed by addition of $400 \mu \mathrm{L}$ of warm HBSS at the basolateral compartments and $200 \mu \mathrm{L}$ of $100 \mu \mathrm{g} / \mathrm{mL}$ FITC-conjugated Dextran 4000 solution in HBSS to apical compartments. After $3 \mathrm{~h}$, Dextran-FITC flux across monolayers was measured by transferring $300 \mu \mathrm{L}$ of basolateral HBSS to a 96-well plate (corning) and measuring fluorescence using an ELISA reader (Tecan Infinite M200) at excitation and emission wavelengths of 492 and 518, respectively.

2.5. Flow Cytometry. Annexin-V binding assay was used to observe early apoptotic changes in Caco-2 cells. Caco-2 monolayers were grown in 24-well culture plates and coincubated with Blastocystis ST-7 or ST-4 for 3 h. Monolayers were then washed with PBS twice and then were trypsinized with $0.25 \%$ trypsin-EDTA, resuspended, and collected. AnnexinV-FITC apoptosis detection kit (BioVision) was used according to manufacturer's instructions. Propidium iodide (PI) was used to exclude necrotic cells. After cell staining, samples were analyzed using a flow cytometer (DakoCytomation; Cyan LX) at $488 \mathrm{~nm}$ excitation wavelength, with a $515 \mathrm{~nm}$ band-pass filter for fluorescein detection and a $600 \mathrm{~nm}$ filter for PI detection. The lower right quadrant was defined to represent the apoptotic cells showing annexin-V-FITCpositive and PI-negative staining.

2.6. Immunohistochemistry and Confocal Microscopy. Monolayers were incubated with Blastocystis for $6 \mathrm{~h}$ for immunohistochemical detection of ZO-1 rearrangements in Caco2 cells. After coincubation, monolayers were washed twice and fixed with $2 \%(\mathrm{w} / \mathrm{v})$ formaldehyde in PBS. Cells were then washed and incubated overnight with $1000 \times$ dilution of primary antibody against ZO-1 tight junction protein (Sigma; $1: 1000$ in PBS) at $4^{\circ} \mathrm{C}$. Monolayers were washed twice with PBS and incubated for $1 \mathrm{~h}$ with Cy3-tagged secondary antibody, followed by another round of washing. For DNA staining assay, monolayers were treated with parasites for $24 \mathrm{~h}$, washed, and fixed as described above. After fixation, monolayers were washed and incubated with $10 \mu \mathrm{g} / \mathrm{mL}$ of cell-permeable DNA-stain Hoechst (Invitrogen) for $10 \mathrm{~min}$ and washed again. All monolayers were mounted on a glass slide using fluorescence mounting media (VECTASHIELD) before being observed under a confocal microscope (Olympus BX60; Olympus, Japan). The ImageJ software was used for image analysis.

2.7. Western Blots. For western blot analysis, Caco-2 monolayers were grown on 6-well cell-culture plates (corning) until $100 \%$ confluency. Monolayers were then incubated with 1 $\times 10^{8}$ parasites $/ \mathrm{mL}$ for 6 and $12 \mathrm{~h}$, respectively, washed with

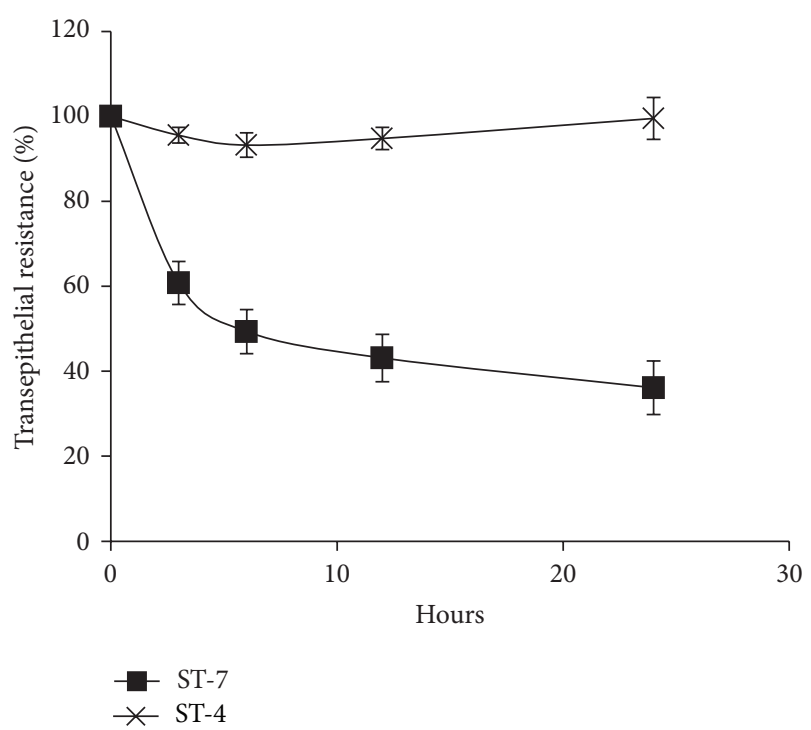

FIgure 1: Time-dependent effect of Blastocystis ST-7 and ST-4 on the transepithelial resistance (TER) of Caco-2 cell monolayers. Confluent monolayers of Caco-2 cells were coincubated with ST7 and ST-4 for the indicated times. TER was then measured as described in Section 2. ST-7 treated monolayers, compared to ST-4 and negative control, showed a significant drop in TER after 3, 6, 12, and $24 \mathrm{~h}(P$ value $<0.01)$. Values are means \pm standard error (error bars) $(n=6)$.

PBS twice, and then scraped and collected. Monolayers were then incubated with RIPA lysis buffer supplemented with protease and phosphatase inhibitors (Pierce). Lysed samples were centrifuged at $21,000 \mathrm{rpm}$ at $4^{\circ} \mathrm{C}$ for 30 minutes. Protein concentration of the supernatant was determined with the $\mathrm{D}_{C}$ Protein Assay (Bio-Rad Laboratories). SDS-PAGE gels (12\% and $15 \%$ Tris-HCL Ready-Gels; Bio-Rad Laboratories) were used to separate total proteins. Proteins were then transferred using a polyvinylidene difluoride (PVDF) membrane (Immobilon-P; Millipore). 5\% of nonfat dry milk in \% TBS$\mathrm{T}$ was then used to block the membranes. After blocking, membranes were incubated with primary antibodies against, caspase 3, caspase 8, caspase 9, or ZO-1 (1:1000; Sigma) overnight at $4^{\circ} \mathrm{C}$. After incubation with primary antibodies, membranes were washed and incubated with HRP-tagged secondary antibodies. Bands were detected using Amersham ECL Plus western blotting detection system (GE Healthcare). Autoradiographic films (Kodak) were then exposed to the membranes and developed on X-ray film processor SRX-101A (Konica Minolta).

2.8. Statistical Analysis. The ANOVA test was used to confirm the statistical significance of our results.

\section{Results}

3.1. Blastocystis ST-7 Decreases Transepithelial Resistance (TER) and Increases Permeability to FITC-Conjugated Dextran in Caco-2 Monolayers. Blastocystis ST-7, but not ST-4, induced a time-dependent drop in Caco-2 TER (Figure 1). 


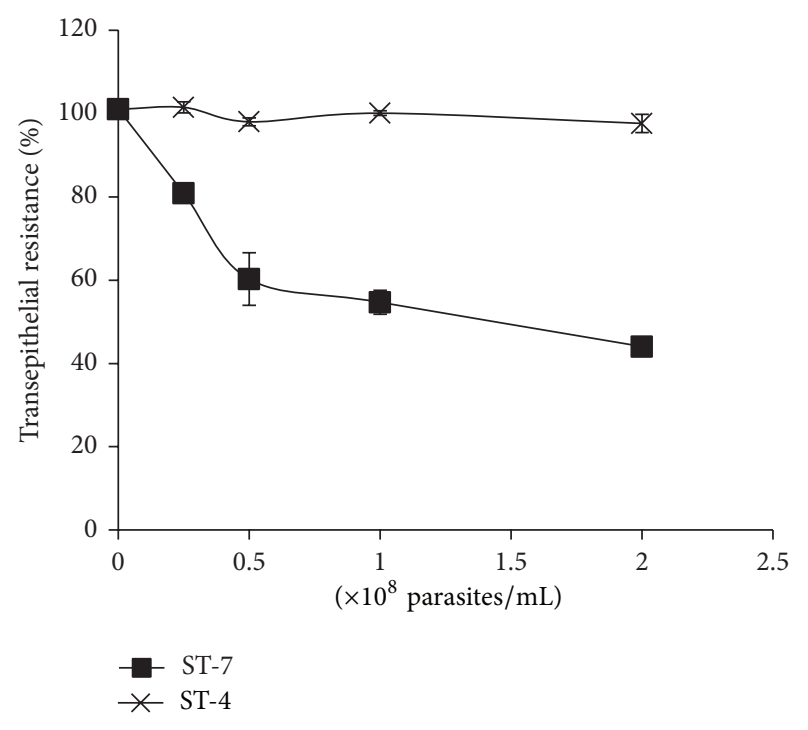

FIGURE 2: Dose-dependent effect of Blastocystis ST-4 and ST-7 on transepithelial resistant (TER) of Caco- 2 cell monolayers. Confluent monolayers were coincubated with varying doses of ST-4 and ST-7 for $24 \mathrm{~h}$. Compared to negative control, Blastocystis ST-7 induced a significant drop in Caco-2 TER at $0.25,0.5,1$, and $2 \times 10^{8}$ parasite $/ \mathrm{mL}$ $(P$ value $<0.01)$. Values are mean \pm standard error (error bars) $(n=$ $6)$.

A significant drop in Caco-2 TER was observed as early as after $3 \mathrm{~h}$ of coincubation $(P$ value $<0.01)$. ST-7 also exhibited a dose-dependent effect on TER (Figure 2). The minimum dose of $0.25 \times 10^{8} / \mathrm{mL}$ of ST-7 induced a significant drop in epithelial resistance (Figure 2). In order to confirm the parasite-mediated epithelial dysfunction suggested by drop in TER, we measured the flux of Dextran-FITC probe across Caco-2 monolayers (Figure 4). As expected, a significant increase in epithelial permeability was observed when $10^{8}$ parasite $/ \mathrm{mL}$ of ST-7 was coincubated with Caco-2 for $24 \mathrm{~h}(P$ value $<0.01)$ (Figure 4$)$. On the other hand ST-4 did not cause a significant change in TER (Figures 1, 2, and 3) neither at the highest parasite dose $\left(2 \times 10^{8}\right)$ (Figure 2) nor after the longest coincubation period $(24 \mathrm{~h})$ (Figure 1 ). ST-4 did not induce increase in epithelial permeability to FITC-conjugated Dextran either (Figure 4). This suggests a strain-dependent variation in parasite-mediated Caco-2 barrier disruption.

\subsection{Blastocystis ST-7 Induces Early and Late Apoptotic Changes in Caco-2 Cells}

(i) PS-Flipping Indicated by Annexin-FITC Binding. One of the early indicators of apoptosis is the flipping of phosphatidylserine (PS) molecules from the inner to outer leaflet of the plasma membrane. A 35- to 36-kDa molecule, annexin$\mathrm{V}$, binds to PS with high specificity in the presence of calcium. Viable, apoptotic, and necrotic cells can be distinguished when FITC conjugated-annexin- $\mathrm{V}$ is used in conjunction

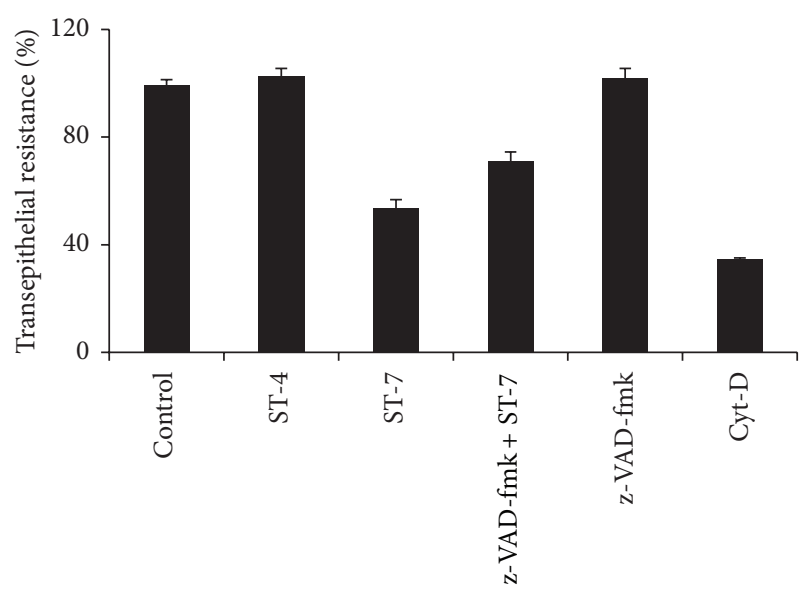

FIGURE 3: Effects of caspase inhibition on Blastocystis ST-7-induced decrease in transepithelial resistance (TER) of Caco-2 monolayers. Confluent monolayers of Caco-2 cells were coincubated for $24 \mathrm{~h}$ with ST-7 after pretreatment of cells with broad-spectrum caspase inhibitor z-VAD-fmk. Thereafter, TER was measured as described in Section 2. Pretreatment of Caco-2 cells with caspase inhibitor considerably rescued these cells from Blastocystis ST-7-induced effect $(P$ value $<0.01)$. Cytochalasin D (Cyt-D) was used as a positive control in decreasing TER. Values are means \pm standard error (error bars) $(n=6)$.

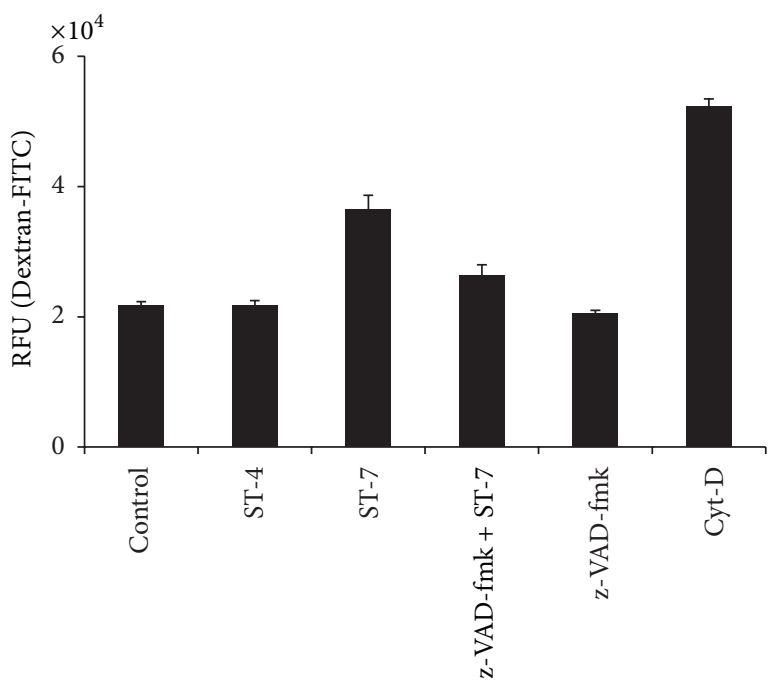

FIgURE 4: Flux measurement with FITC-conjugated Dextran. Confluent monolayers of Caco-2 cells were coincubated for $24 \mathrm{~h}$ with Blastocystis ST-4 or ST-7. Some monolayers were coincubated with ST-7 after pretreatment of cells with the broad-spectrum caspase inhibitor Z-VAD-fmk. Permeability was determined by measurement of Dextran-FITC fluxes across the monolayer as described in Section 2. A significant increase in the epithelial permeability can be noticed after incubation with ST-7, compared to negative control and ST-4 coincubation $(P$ value $<0.01)$. Pretreatment of Caco- 2 cells with caspase inhibitor significantly rescued these cells from Blastocystis-induced effect on permeability. Cytochalasin D (Cyt-D) was used as a positive control in inducing permeability increase. Values are means \pm standard error (error bars) $(n=6)$. 

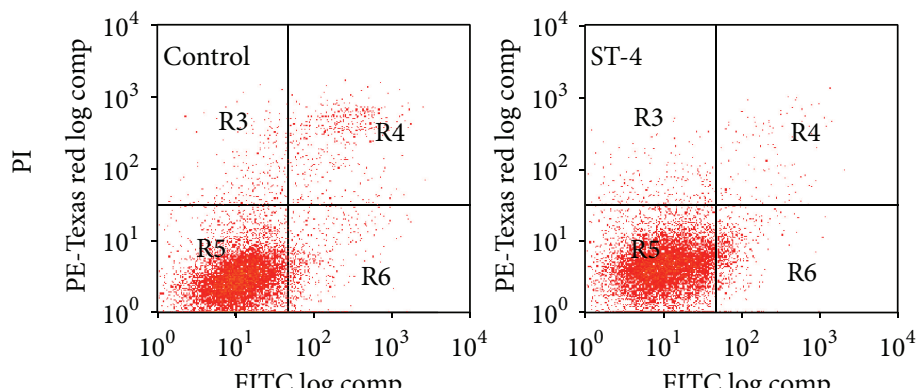

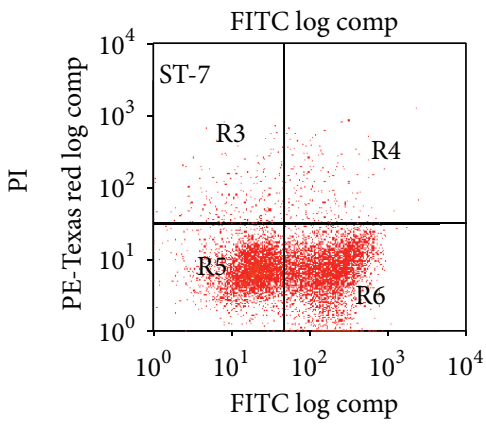

Annexin-V-FITC

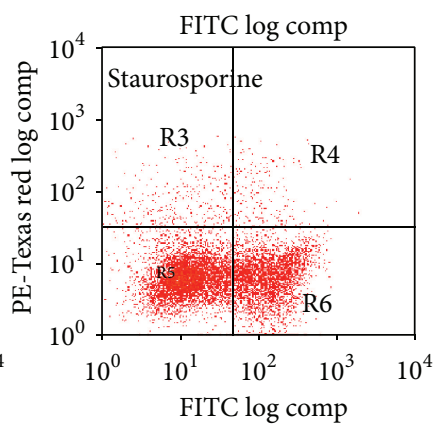

Annexin-V-FITC

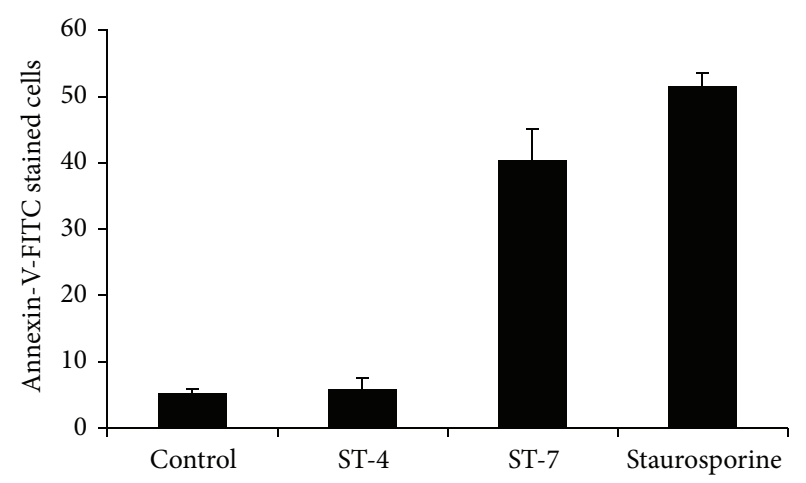

FIGURE 5: Flow cytometry analysis of annexin-V-FITC and propidium iodide staining. Representative dot plots of Caco-2 cells preincubated with culture media as negative control, Blastocystis ST-7, ST-4, and $0.5 \mu \mathrm{M}$ staurosporine as positive control. $2 \times 10^{4}$ cells were analysed in each sample. Values represent mean \pm standard error (error bars; $n=3$ ). Caco-2 cells interacting with ST-7 exhibited significantly higher percentage of annexin- $\mathrm{V}^{+}$and $\mathrm{PI}^{-}$cells (lower right quadrant) compared to cells coincubated with ST-4 or culture media only $(P$ value $<$ $0.01)$. Values are means \pm standard error (error bars) $(n=6)$.

with PI. The lower right quadrants of the dot plots represent the apoptotic cell population, positive for annexin binding but PI negative (Figure 5). Upper quadrants represent necrotic cells due to permeability to PI (Figure 5). After interaction with Blastocystis ST-7, Caco-2 cells exhibited a significant rise in percentage of apoptotic cells compared to negative control and those interacting with ST-4 $(P$ value $<$ 0.01 ) (Figure 5). These findings are in agreement with a straindependent pathogenicity of the parasite.

(ii) Nuclear Fragmentation Indicated by Hoechst Staining [41]. One of the most distinctive features of apoptosis is morphological change in the nucleus, easily observed under florescence microscopy (Figure 6). After $24 \mathrm{~h}$ interaction with Blastocystis ST-7, nuclei of the Caco-2 cells exhibited nuclear condensation and fragmentation (Figure 6) typical of apoptotic cells. Significantly higher number of apoptotic cells was observed in membranes interacting with ST-7 compared to those with ST-4 $(P$ value $<0.01)$ and negative control $(P$ value $<0.01$ ) (Figure 6).

\subsection{Blastocystis ST-7 Induces Caspases 3 and 9 Activation in} Caco-2 Cells. Caspases are proenzymes, which are activated by cleavage into active fragments in apoptotic cells. In this study, western blot analysis revealed that Blastocystis ST-7 coincubation with Caco-2, resulted in cleavage of Caco-2 caspases 3 and 9 (Figure 7). No cleavage of caspase 8 was observed even after $12 \mathrm{~h}$ interaction with ST-7 (Figure 7) suggesting that Blastocystis ST-7 induces Caco-2 programmed cell death by activation of the intrinsic apoptotic pathway. ST4 , as expected, did not activate any of the three caspases tested in this study (Figure 7). 

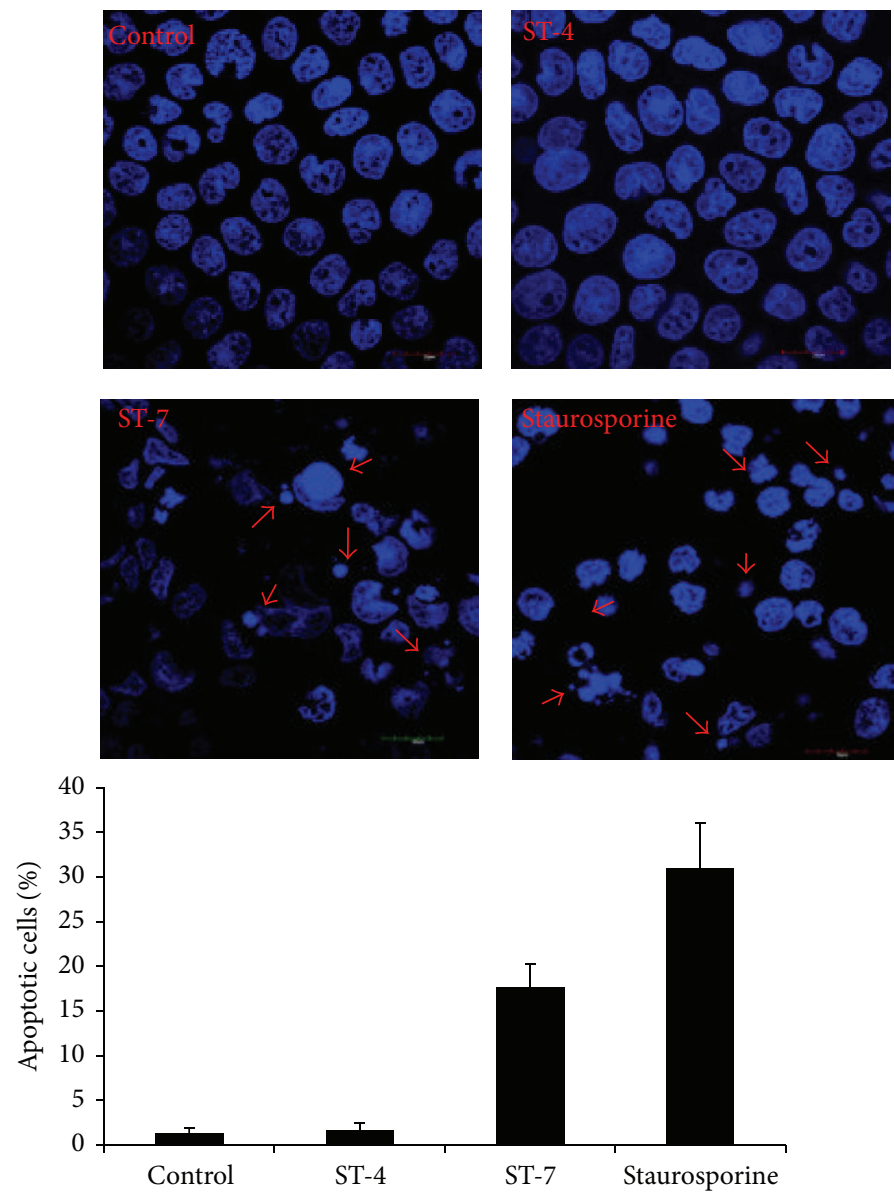

FIGURE 6: Representative fluorescence micrographs showing apoptosis of Caco-2 cells after DAPI staining. Cells were grown on glass coverslips and incubated for $24 \mathrm{~h}$ with culture media, ST-4, ST-7, or $0.5 \mu \mathrm{M}$ staurosporine as positive control. Cells coincubated with ST7 and staurosporine exhibit nuclear fragmentation and condensation (arrow) typical of apoptotic cells. Histogram represents percentage of apoptotic cells after DAPI fluorescence assay. Caco-2 monolayers coincubated with ST-7 exhibited significantly higher percentage of apoptosis $(P$ value $<0.05)$ compared to ST- 4 and negative control. Values are means \pm standard error $(n=6$ per group). For each sample, $\sim 100$ cells were counted at $1000 \mathrm{x}$ magnification.

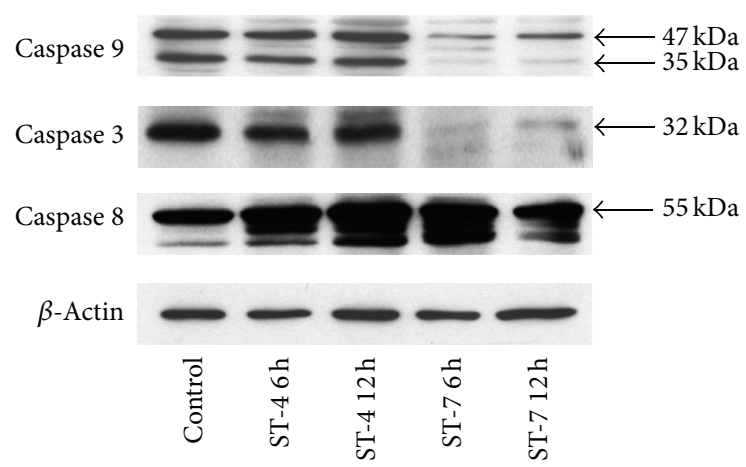

Figure 7: Western blot analysis of caspase activation (cleavage) in Caco-2 cells. Caco-2 cells were grown on cell-culture plates and harvested after coincubation with Blastocystis ST-4 and ST-7 for 6 and $12 \mathrm{~h}$, as described in Section 2. Interaction with ST-7 resulted in loss of caspase 3 and caspase 9 bands suggesting activation, while caspase 8 remained unchanged. ST-4 did not cause any cleavage of caspases in Caco-2. 

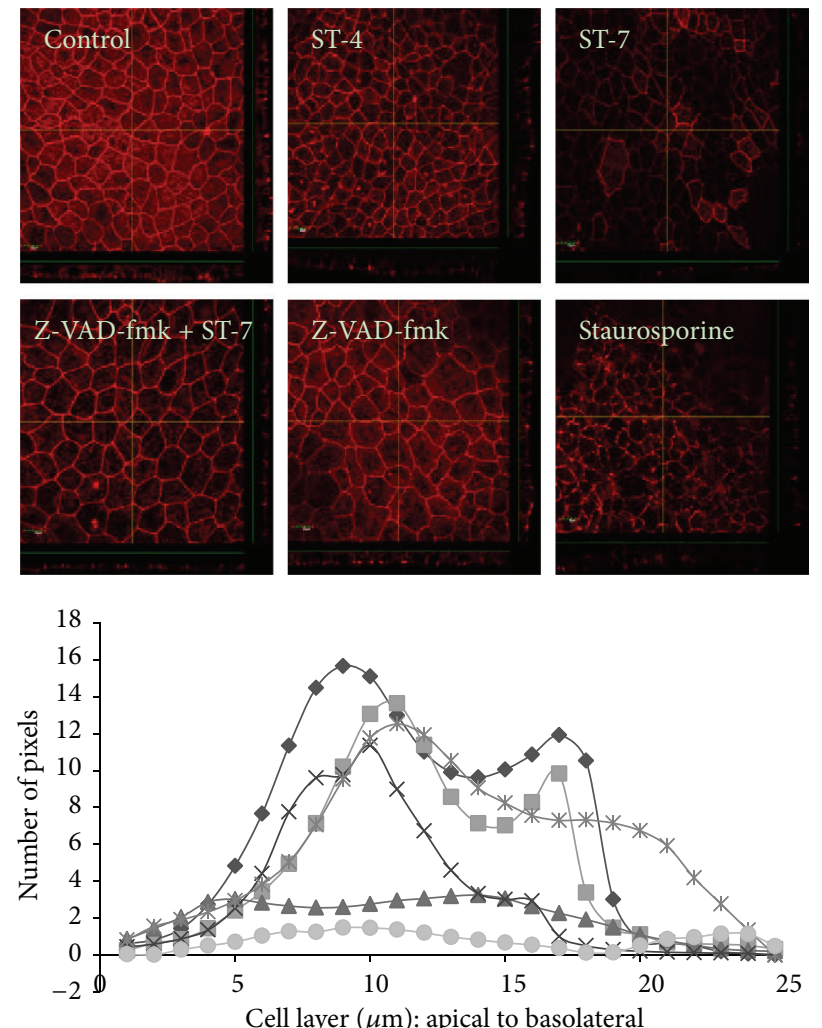

$$
\begin{aligned}
& - \text { Control }^{*} \quad * \text { z-VAD-fmk }+ \text { ST-7** } \\
& - \text { ST-4 number } \quad * \text { z-VAD-fmk } \\
& - \text { ST-7 }{ }^{*} \text {, number, }{ }^{* *} \rightarrow \text { Staurosporine } \\
& { }^{*} \text { Control versus ST-7 }(P \text { value }<0.01) \\
& \text { \#ST-4 versus ST-7 }(P \text { value }<0.01) \\
& { }^{* *} \text { ST-7 versus z-VAD-fmk + ST-7 }(P \text { value }<0.01)
\end{aligned}
$$

FIGURE 8: (a) Representative confocal micrographs illustrating ZO-1 distribution in Caco-2 monolayers. Monolayers were grown to confluence on poly-L-lysine treated coverslips. Caco- 2 cells were then coincubated for $6 \mathrm{~h}$ with either Blastocystis ST- 4 or ST-7. Some monolayers were treated with broad-spectrum caspase inhibitor Z-VAD-fmk before coincubation with ST-7. Normal culture media and $0.5 \mu \mathrm{M}$ staurosporine were used as negative and positive controls, respectively. Compared to negative control, ST-7 treatment resulted in obvious reduction in ZO-1 apical localization in Caco-2 cell line. ST-4 did not alter ZO-1 integrity. Pretreatment with z-VAD-fmk rescued ST-7-induced ZO-1 changes in the epithelium. (b) Quantification of ZO-1 staining is shown as graphs. Each cell layer (1-25) corresponds to series of images from Z-stack sections taken at $1 \mu \mathrm{m}$ thickness through the cell monolayer shown in Figure $8 . X$-axis illustrates cell layers from apical to basolateral. $Y$-axis illustrates the number of pixels present over the entire area of image. Monolayers interacting with ST-7 and treated with staurosporine resulted in marked reduction in number of pixels in cell layers representing apical region, compared to ST-4 treated epithelium and normal control. Pretreatment of epithelium with broad-spectrum caspase inhibitor, z-VAD-fmk, resulted in inhibition of ST-7-induced ZO-1 changes in the monolayer. Results shown are mean of 4 separate Z-stacks for each treatment. (magnification: 600x).

3.4. Blastocystis ST-7 Induces Caspase-Dependent ZO-1 Rearrangement in Caco-2. Compromise of epithelial barrier function, as observed in this study, is often associated with alterations of tight junction proteins. In this study, we observed that Blastocystis ST-7 induced an alteration in tight junction protein complex in conjunction with epithelial barrier dysfunction (Figures 8 and 9). Confocal micrographs suggest that exposure of Caco-2 monolayer to ST-7 induced a significant drop in anti-ZO-1 antibody binding to the apical junctional ring of epithelium $(P$ value $<0.01)$ (Figure 8$)$. ST4 and negative control, on the other hand, had no effect on epithelial ZO-1 (Figure 8). Furthermore, western blot analysis showed that interaction with ST-7 resulted in a loss of Caco-2 ZO- 1 band at $250 \mathrm{kDA}$ (Figure 9). Interaction with ST- 4 and negative control had no effect on ZO-1 distribution in Caco-2 (Figures 8 and 9). Interestingly, inhibition of host caspases by Z-VAD-fmk prevented ST-7-induced ZO-1 changes (Figures 8 and 9).

3.5. Inhibition of Caco-2 Caspases Prevented Blastocystis ST-7Induced Epithelial Barrier Dysfunction. It has been reported previously that host epithelial dysfunction induced by luminal parasites is caused by increased apoptosis in enterocytes. In this study, we observed that inhibition of host caspases by 


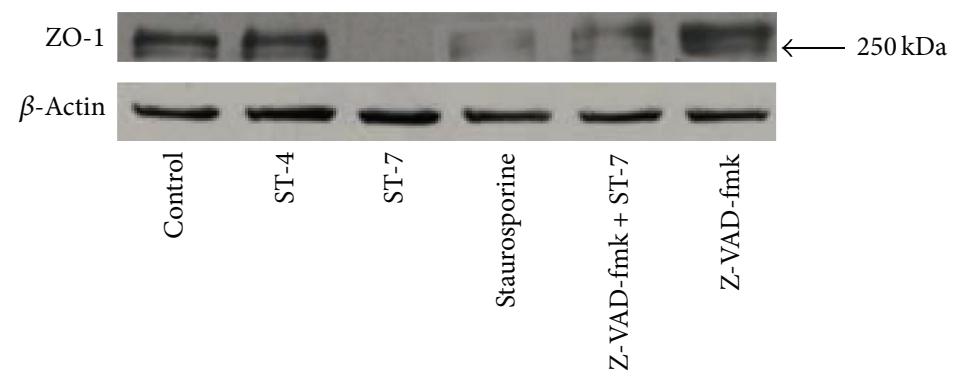

FIGURE 9: Western blot analysis of ZO-1 integrity in Caco-2 epithelium. Monolayers were grown on cell-culture plates and harvested after coincubation with Blastocystis ST-4, ST-7, normal growth media, and $0.5 \mu \mathrm{M}$ staurosporine. Monolayers were also treated with broadspectrum caspase inhibitor z-VAD-fmk before incubation with ST-7. After coincubation with ST-7 and staurosporine for $6 \mathrm{~h}$, loss of ZO-1 band was observed. The ZO-1 band remained unchanged in untreated Caco-2 cells and those interacting with ST-4. Caspase inhibition resulted in rescue of ST-7-induced loss of ZO-1.

broad-spectrum caspase inhibitor, z-VAD-fmk, significantly prevented ST-7-induced TER drop $(P$ value $<0.01)$ (Figure 3 ) and inhibited parasite-induced increase in permeability $(P$ value $<0.01$ ) (Figure 4 ) of Caco-2 monolayers. These findings (Figures 3 and 4), in conjunction with inhibition of ST-7induced ZO-1 alteration (Figures 8 and 9) by z-VAD-fmk, suggested a role of parasite-induced apoptosis in epithelial barrier dysfunction.

\section{Discussion}

In this study, using two clinically relevant Blastocystis isolates, we reported that a Blastocystis ST-7 isolate recovered from a human patient-induced epithelial barrier dysfunction in Caco-2 human epithelial cell-line, while a ST-4 isolate recovered from rat host did not induce enterocyte pathology. The intestinal epithelium serves as the body's first line of defense against luminal contents comprising of a diverse flora including pathogenic and nonpathogenic prokaryotes and eukaryotes [33]. Intestinal epithelial cells or enterocytes regulate the back and forth flow of contents between the gut lumen and subepithelial tissue [33]. Dysfunction of this barrier is the cause of a wide range of human diseases [28]. Blastocystis-induced compromise of human epithelial barrier, observed in our study, reinforces its status as a human pathogen.

We are also reporting for the first time that Blastocystis causes apoptosis in human epithelial cells. Apoptosis is a preprogrammed mechanism of clearing up unwanted cells by the body [42] without overtly stimulating a host immune response [36]. Cells undergo apoptosis even under physiological conditions, but several pathogens including parasites have evolved mechanisms to disrupt this machinery, by either upregulating [38, 43] or downregulating [44] it for their survival in the host. Blastocystis ST-7 induced both early and late hallmarks of apoptosis, that is, PSexternalization and nuclear fragmentation, respectively, in human enterocytes. Increased enterocyte apoptosis in some cases is also proposed to be a host response to infection by increasing the cell turnover in order to rid the body of the infected cells [45]. Mucosal sloughing reported during Blastocystis infections [46] might be a result of this increased turnover. Anti-inflammatory effects of apoptosis are also well recognized [47]. The relatively moderate level of apoptosis induced by Blastocystis in intestinal epithelium is similar to the programmed cell death of enterocytes when exposed to Cryptosporidium [48] and Giardia [38]. It is suggested that upregulation of host cell apoptosis might be a reason for lack of overt host inflammatory response during parasitic infections $[38,48]$ and might assist them in colonizing the hostile host environment. Although further investigation is needed in this area, the absence of obvious gut inflammatory changes in Blastocystis-infected hosts might be due to the ability of the parasite to downregulate host inflammatory response by induction of enterocyte apoptosis.

This is also the first study reporting that Blastocystis induces epithelial apoptosis by activation of the intrinsic pathway. Caspase 3 activation lies at the center of the caspase-mediated apoptosis [42]. It is preceded by activation of either intrinsic pathway, involving mitochondrial injury and caspase 9 activation, or extrinsic pathway due to Fas/FasL receptor-mediated caspase 8 activation. Parasites have evolved complex mechanisms to activate epithelial caspase 3. Giardia activates extrinsic as well as intrinsic apoptotic pathways [49]. Entamoeba on the other hand does not require either caspase 8 or caspase 9 for enterocyte caspase 3 activation [50]. In an earlier study, we reported that Blastocystis ST-4 induced rat epithelial apoptosis by caspase 3 activation [39]. There is no data available suggesting a similar outcome in human epithelium. Upstream pathways involved in caspase 3 activation by the parasite are not known either. In this study, the rodent strain had no effect on Caco-2 cell line, but ST-7, isolated from human host, induced caspase 3 activation. Blastocystis unlike Giardia or Entamoeba only activated caspase 9. A recent study suggested the involvement of Rho kinases in selective activation of caspase 9, leading to apoptosis [51]. A role of Rho kinase has been suggested in Blastocystis-induced breakdown of host epithelial barrier function and cytoskeletal rearrangement [22]. Although more data is required, selective activation of caspase 9 by Blastocystis in this study might be a result of epithelial Rho kinase modulation by the parasite. Further understanding of the unique cellular mechanisms employed by Blastocystis to induce host cell apoptosis might help us 
to develop targeted therapeutics to prevent parasite-induced host pathology.

Tight junctions are key regulators of epithelial barrier function [33, 52]. ZO-1 is an important component of apical junctional complex, anchoring tight junctions to actin cytoskeleton [33]. Our data shows that Blastocystis ST-7 induces rearrangement of ZO-1 in intestinal epithelium. Several parasites are known to cause ZO-1 alterations by a wide array of mechanisms $[33,53,54]$. Acanthamoeba activates Rho/Rho-kinase pathway [53], whereas Giardia utilizes myosin light chain kinase [54] and caspases [38] to induce changes in ZO-1 organization. Apoptosis also plays a diverse role in the modulation of epithelial barrier function and tight junction reorganization. On one hand, enterocyte apoptosis ensures that epithelial barrier remains sealed [52], while in other cases induction of apoptosis is employed by pathogens to increase epithelial permeability [38] and cause host pathology [37]. Inhibition of host caspases resulted in prevention of Giardia-induced modulation of epithelial permeability and ZO-1 organization [38] while, inhibition of caspase-mediated apoptosis in enteropathogenic E. coli (EPEC) infections did not prevent the epithelial ZO-1 alterations [55]. In a recent study with rodent epithelium, pretreatment of host epithelium with pan-caspase inhibitor $\mathrm{z}$-VAD-fmk did not rescue Blastocystis ST-4-induced epithelial barrier dysfunction [39]. In the current study, z-VAD-fmk treatment of epithelium significantly inhibited Blastocystis ST-7-induced epithelial barrier compromise. Parasite-induced ZO-1 alteration was also prevented significantly by host caspase inhibition, reiterating that role of enterocyte apoptosis in parasite- induced epithelial barrier dysfunction. Interestingly, changes in ZO-1 were also shown to be prevented by Rho kinase [22], again raising the question of the role of Rho kinase in caspasemediated apoptosis.

We also observed for the first time a strain-dependent variation in Blastocystis-induced epithelial barrier compromise. Blastocystis ST-4 did not induce an increase in epithelial permeability, enterocyte apoptosis, or ZO-1 rearrangement in Caco-2 cells. We have also similarly observed that ST7 and not ST-4 induced apoptosis in HT-29 cells, another transformed human intestinal epithelial cell line (results not shown). Several studies have suggested strain-dependent differences in Blastocystis virulence [8, 25]. Strain-to-strain variation in virulence is not unique to Blastocystis and it has been observed in intestinal parasites such as Giardia, Cryptosporidium, and Entamoeba [38, 56, 57], providing a plausible explanation for the large number of asymptomatic carriers of these pathogens $[58,59]$. This might also explain frequent reports of asymptomatic Blastocystis carriers. In a recent study [26], we reported that Blastocystis exhibits a strain-dependent variation in the activity of cysteine proteases, potential parasite virulence factors $[22,27]$. ST-7 of the parasite, reported to have higher cysteine protease activity [26], caused epithelial barrier dysfunction in the current study, while ST-4 with comparatively lower cysteine protease activity [26] did not. Although more data is needed, these findings suggest a possible association between parasite cysteine protease activity and its ability to induce epithelial barrier dysfunction. Interestingly, ST-4 caused epithelial barrier dysfunction in rat epithelium [39], suggesting that this strain exhibits host specificity in its ability to induce epithelial barrier compromise, as observed in Cryptosporidium infections [56]. Although human ST-4 infections are commonly associated with intestinal symptoms, its inability to induce epithelial barrier compromise in this study suggests that ST-4 might induce human pathology by some other mechanisms $[60,61]$.

To conclude, this is the first study to show that Blastocystis ST-7, a parasite subtype recovered from a human patient, induced enterocyte-apoptosis by activating caspases 3 and 9 , suggesting the involvement of the intrinsic apoptotic pathway in pathogenesis. We also showed that this cytopathic human isolate of Blastocystis (ST-7) caused rearrangement of ZO1 protein. Inhibition of host caspases prevented parasiteinduced epithelial barrier dysfunction as well as ZO-1 rearrangement, suggesting the role of caspase-dependent enterocyte apoptosis in host epithelial barrier dysfunction induced by Blastocystis. Furthermore, the inability of rodent subtype ST-4 to induce any changes in Caco-2 provides evidence of host specificity and strain dependency in Blastocystisinduced human epithelial pathology. The strain-to-strain variation in parasite virulence is a plausible explanation for the large number asymptomatic human carriers of Blastocystis.

\section{Conflict of Interests}

The authors declare that there is no conflict of interests regarding the publication of this paper.

\section{Authors' Contribution}

Zhaona $\mathrm{Wu}$ and Haris Mirza contributed equally to the paper.

\section{References}

[1] K. S. Tan, "New insights on classification, identification, and clinical relevance of Blastocystis spp.", Clinical Microbiology Reviews, vol. 21, no. 4, pp. 639-665, 2008.

[2] C. Noël, F. Dufernez, D. Gerbod et al., "Molecular phylogenies of Blastocystis isolates from different hosts: implications for genetic diversity, identification of species, and zoonosis," Journal of Clinical Microbiology, vol. 43, no. 1, pp. 348-355, 2005.

[3] K. S. Tan, H. Mirza, J. D. W. Teo, B. Wu, and P. A. MacAry, "Current views on the clinical relevance of Blastocystis spp." Current Infectious Disease Reports, vol. 12, no. 1, pp. 28-35, 2010.

[4] P. D. Scanlan and C. R. Stensvold, "Blastocystis: getting to grips with our guileful guest," Trends in Parasitology, vol. 29, no. 11, pp. 523-529, 2013.

[5] V. Mehraj, J. Hatcher, S. Akhtar, G. Rafique, and M. A. Beg, "Prevalence and factors associated with intestinal parasitic infection among children in an urban slum of Karachi," PLoS ONE, vol. 3, no. 11, Article ID e3680, 2008.

[6] A. Kurniawan, T. Karyadi, S. W. Dwintasari et al., "Intestinal parasitic infections in HIV/AIDS patients presenting with diarrhoea in Jakarta, Indonesia," Transactions of the Royal Society of Tropical Medicine and Hygiene, vol. 103, no. 9, pp. 892-898, 2009. 
[7] Y. Taşova, B. Şahin, S. Koltaş, and S. Paydaş, "Clinical significance and frequency of Blastocystis hominis in Turkish patients with hematological malignancy," Acta Medica Okayama, vol. 54, no. 3, pp. 133-136, 2000.

[8] C. R. Stensvold, H. C. Lewis, A. M. Hammerum et al., "Blastocystis: unravelling potential risk factors and clinical significance of a common but neglected parasite," Epidemiology and Infection, vol. 137, no. 11, pp. 1655-1663, 2009.

[9] J. Yakoob, W. Jafri, M. A. Beg et al., "Blastocystis hominis and Dientamoeba fragilis in patients fulfilling irritable bowel syndrome criteria," Parasitology Research, vol. 107, no. 3, pp. 679-684, 2010.

[10] M. E. Ramirez-Miranda, R. Hernandez-Castellanos, E. LopezEscamilla et al., "Parasites in Mexican patients with irritable bowel syndrome: a case-control study," Parasites and Vectors, vol. 3, no. 1, p. 96, 2010.

[11] P. Poirier, I. Wawrzyniak, C. P. Vivares, F. Delbac, and H. El Alaoui, "New insights into Blastocystis spp.: a potential link with irritable bowel syndrome," PLoS Pathog, vol. 8, Article ID e1002545, 2012.

[12] D. M. Hameed, O. M. Hassanin, and N. M. Zuel-Fakkar, "Association of Blastocystis hominis genetic subtypes with urticaria," Parasitology Research, vol. 108, no. 3, pp. 553-560, 2011.

[13] C. Vogelberg, C. R. Stensvold, S. Monecke et al., "Blastocystis sp. subtype 2 detection during recurrence of gastrointestinal and urticarial symptoms," Parasitology International, vol. 59, no. 3, pp. 469-471, 2010.

[14] M. R. Sohail and P. R. Fischer, "Blastocystis hominis and travelers," Travel Medicine and Infectious Disease, vol. 3, no. 1, pp. 33-38, 2005.

[15] H. Mirza, Z. Wu, F. Kidwai, and K. S. W. Tan, "A metronidazoleresistant isolate of Blastocystis spp. Is susceptible to nitric oxide and downregulates intestinal epithelial inducible nitric oxide synthase by a novel parasite survival mechanism," Infection and Immunity, vol. 79, no. 12, pp. 5019-5026, 2011.

[16] S. Chandramathi, K. Suresh, and U. R. Kuppusamy, "Elevated levels of urinary hyaluronidase in humans infected with intestinal parasites," Annals of Tropical Medicine and Parasitology, vol. 104, no. 5, pp. 449-452, 2010.

[17] S. Chandramathi, K. G. Suresh, A. A. Mahmood, and U. R. Kuppusamy, "Urinary hyaluronidase activity in rats infected with Blastocystis hominis-evidence for invasion?" Parasitology Research, vol. 106, no. 6, pp. 1459-1463, 2010.

[18] K.-C. Hu, C.-C. Lin, T.-E. Wang, C.-Y. Liu, M.-J. Chen, and W.H. Chang, "Amoebic liver abscess or is it?" Gut, vol. 57, no. 5, pp. 627-683, 2008.

[19] W. D. Patino, D. Cavuoti, S. K. Banerjee, K. Swartz, R. Ashfaq, and T. Gokaslan, "Cytologic diagnosis of Blastocystis hominis in peritoneal fluid: a case report," Acta Cytologica, vol. 52, no. 6, pp. 718-720, 2008.

[20] A. Alexeieff, "Sur la nature des formations dites, 'Kystes de Trichomonas intestinalis,' Comptes Rendus des Séances et Mémoires de la Société de Biologie, vol. 71, pp. 296-298, 1911.

[21] E. Brumpt, "Blastocystis hominis n sp. et formes voisines," Bulletin de la Société de Pathologie Exotique, vol. 5, pp. 725-730, 1912.

[22] H. Mirza, Z. Wu, J. D. Teo, and K. S. Tan, "Statin pleiotropy prevents rho kinase-mediated intestinal epithelial barrier compromise induced by Blastocystis cysteine proteases," Cellular Microbiology, vol. 14, no. 9, pp. 1474-1484, 2012.
[23] H. Mirza and K. S. Tan, "Clinical aspects of Blastocystis infections: advancements amidst controversies," in Blastocystis: Pathogen or Passenger? H. Mehlhorn, K. S. Tan, and H. Yoshikawa, Eds., pp. 65-84, Springer, Berlin, Germany, 2012.

[24] A. Iguchi, A. Ebisu, S. Nagata et al., "Infectivity of different genotypes of human Blastocystis hominis isolates in chickens and rats," Parasitology International, vol. 56, no. 2, pp. 107-112, 2007.

[25] E. Hussein, A. Hussein, M. Eida, and M. Atwa, "Pathophysiological variability of different genotypes of human Blastocystis hominis Egyptian isolates in experimentally infected rats," Parasitology Research, vol. 102, no. 5, pp. 853-860, 2008.

[26] H. Mirza and K. S. Tan, "Blastocystis exhibits inter- and intrasubtype variation in cysteine protease activity," Parasitology Research, vol. 104, no. 2, pp. 355-361, 2009.

[27] M. Puthia, A. Vaithilingam, J. Lu, and K. S. W. Tan, "Degradation of human secretory immunoglobulin a by Blastocystis," Parasitology Research, vol. 97, no. 5, pp. 386-389, 2005.

[28] J. R. O'Hara and A. G. Buret, "Mechanisms of intestinal tight junctional disruption during infection," Frontiers in Bioscience, vol. 13, no. 18, pp. 7008-7021, 2008.

[29] H. J. Epple, T. Schneider, H. Troeger et al., "Impairment of the intestinal barrier is evident in untreated but absent in suppressively treated HIV-infected patients," Gut, vol. 58, no. 2, pp. 220-227, 2009.

[30] T. Matysiak-Budnik, B. Coffin, A. Lavergne-Slove, J. Sabate, F. Mégraud, and M. Heyman, "Helicobacter pylori increases the epithelial permeability to a food antigen in human gastric biopsies," American Journal of Gastroenterology, vol. 99, no. 2, pp. 225-232, 2004.

[31] H. Troeger, H. Epple, T. Schneider et al., "Effect of chronic Giardia lamblia infection on epithelial transport and barrier function in human duodenum," Gut, vol. 56, no. 3, pp. 328-335, 2007.

[32] S. Rawal, S. Majumdar, and H. Vohra, "Activation of MAPK kinase pathway by Gal/GalNAc adherence lectin of E. histolytica: gateway to host response," Molecular and Cellular Biochemistry, vol. 268, no. 1-2, pp. 93-101, 2005.

[33] J. R. Turner, "Intestinal mucosal barrier function in health and disease," Nature Reviews Immunology, vol. 9, no. 11, pp. 799-809, 2009.

[34] H. L. Nielsen, H. Nielsen, T. Ejlertsen et al., "Oral and fecal Campylobacter concisus strains perturb barrier function by apoptosis induction in HT-29/B6 intestinal epithelial cells," PLoS ONE, vol. 6, no. 8, Article ID e23858, 2011.

[35] A. C. Chin, D. A. Teoh, K. G.-E. Scott, J. B. Meddings, W. K. Macnaughton, and A. G. Buret, "Strain-dependent induction of enterocyte apoptosis by Giardia lamblia disrupts epithelial barrier function in a caspase-3-dependent manner," Infection and Immunity, vol. 70, no. 7, pp. 3673-3680, 2002.

[36] Y. Gao, J. M. Herndon, H. Zhang, T. S. Griffith, and T. A. Ferguson, "Antiinflammatory effects of CD95 ligand (FasL)induced apoptosis," Journal of Experimental Medicine, vol. 188, no. 5, pp. 887-896, 1998.

[37] S. M. Becker, K. Cho, X. Guo et al., "Epithelial cell apoptosis facilitates Entamoeba histolytica infection in the gut," American Journal of Pathology, vol. 176, no. 3, pp. 1316-1322, 2010.

[38] A. Chin, D. Teoh, K. Scott, J. B. Meddings, W. K. Macnaughton, and A. G. Buret, "Strain-dependent induction of enterocyte apoptosis by Giardia lamblia disrupts epithelial barrier function in a caspase-3-dependent manner," Infection and Immunity, vol. 70, no. 7, pp. 3673-3680, 2002. 
[39] M. K. Puthia, S. W. S. Sio, J. Lu, and K. S. W. Tan, “Blastocystis ratti induces contact-independent apoptosis, F-actin rearrangement, and barrier function disruption in IEC-6 cells," Infection and Immunity, vol. 74, no. 7, pp. 4114-4123, 2006.

[40] C. R. Stensvold, H. V. Smith, R. Nagel, K. E. P. Olsen, and R. J. Traub, "Eradication of Blastocystis carriage with antimicrobials: reality or delusion?" Journal of Clinical Gastroenterology, vol. 44, no. 2, pp. 85-90, 2010.

[41] A. M. Petersen, C. R. Stensvold, H. Mirsepasi et al., "Active ulcerative colitis associated with low prevalence of Blastocystis and Dientamoeba fragilis infection," Scandinavian Journal of Gastroenterology, vol. 48, no. 5, pp. 638-639, 2013.

[42] J. Savill, "Recognition and phagocytosis of cells undergoing apoptosis," British Medical Bulletin, vol. 53, no. 3, pp. 491-508, 1997.

[43] C. D. Huston, D. R. Boettner, V. Miller-Sims, and W. A. Petri Jr., "Apoptotic killing and phagocytosis of host cells by the parasite Entamoeba histolytica," Infection and Immunity, vol. 71, no. 2, pp. 964-972, 2003.

[44] T. Yamada, T. Tomita, L. M. Weiss, and A. Orlofsky, “Toxoplasma gondii inhibits granzyme B-mediated apoptosis by the inhibition of granzyme B function in host cells," International Journal for Parasitology, vol. 41, no. 6, pp. 595-607, 2011.

[45] L. J. Cliffe, N. E. Humphreys, T. E. Lane, C. S. Potten, C. Booth, and R. K. Grencis, "Immunology-accelerated intestinal epithelial cell turnover: a new mechanism of parasite expulsion," Science, vol. 308, no. 5727, pp. 1463-1465, 2005.

[46] K. T. Moe, M. Singh, J. Howe et al., "Experimental Blastocystis hominis infection in laboratory mice," Parasitology Research, vol. 83, no. 4, pp. 319-325, 1997.

[47] C. Haslett, "Granulocyte apoptosis and inflammatory disease," British Medical Bulletin, vol. 53, no. 3, pp. 669-683, 1997.

[48] D. F. Mccole, L. Eckmann, F. Laurent, and M. F. Kagnoff, "Intestinal epithelial cell apoptosis following Cryptosporidium parrum infection," Infection and Immunity, vol. 68, no. 3, pp. $1710-1713,2000$.

[49] M. A. Panaro, A. Cianciulli, V. Mitolo et al., "Caspasedependent apoptosis of the HCT-8 epithelial cell line induced by the parasite Giardia intestinalis," FEMS Immunology and Medical Microbiology, vol. 51, no. 2, pp. 302-309, 2007.

[50] C. D. Huston, E. R. Houpt, B. J. Mann, C. S. Hahn, and W. A. Petri Jr., "Caspase 3-dependent killing of host cells by the parasite Entamoeba histolytica," Cellular Microbiology, vol. 2, no. 6, pp. 617-625, 2000.

[51] D. P. Del Re, S. Miyamoto, and J. H. Brown, "RhoA/Rho kinase up-regulate Bax to activate a mitochondrial death pathway and induce cardiomyocyte apoptosis," Journal of Biological Chemistry, vol. 282, no. 11, pp. 8069-8078, 2007.

[52] A. M. Marchiando, L. Shen, W. V. Graham et al., "The epithelial barrier is maintained by in vivo tight junction expansion during pathologic intestinal epithelial shedding," Gastroenterology, vol. 140, no. 4, pp. e1201-e1218, 2011.

[53] N. A. Khan and R. Siddiqui, "Acanthamoeba affects the integrity of human brain microvascular endothelial cells and degrades the tight junction proteins," International Journal for Parasitology, vol. 39, no. 14, pp. 1611-1616, 2009.

[54] K. G. Scott, J. B. Meddings, D. R. Kirk, S. P. LeesMiller, and A. G. Buret, "Intestinal infection with Giardia spp. reduces epithelial barrier function in a myosin light chain kinase-dependent fashion," Gastroenterology, vol. 123, no. 4, pp. 1179-1190, 2002.
[55] A. Buret, M. E. Olson, D. Grant Gall, and J. A. Hardin, "Effects of orally administered epidermal growth factor on enteropathogenic Escherichia coli infection in rabbits," Infection and Immunity, vol. 66, no. 10, pp. 4917-4923, 1998.

[56] A. Hashim, G. Mulcahy, B. Bourke, and M. Clyne, "Interaction of Cryptosporidium hominis and Cryptosporidium parvum with primary human and bovine intestinal cells," Infection and Immunity, vol. 74, no. 1, pp. 99-107, 2006.

[57] P. Davis, J. Schulze, and S. L. Stanley Jr., "Transcriptomic comparison of two Entamoeba histolytica strains with defined virulence phenotypes identifies new virulence factor candidates and key differences in the expression patterns of cysteine proteases, lectin light chains, and calmodulin," Molecular and Biochemical Parasitology, vol. 151, no. 1, pp. 118-128, 2007.

[58] J. Yakoob, Z. Abbas, M. A. Beg et al., "Prevalences of Giardia lamblia and Cryptosporidium parvum infection in adults presenting with chronic diarrhoea," Annals of Tropical Medicine and Parasitology, vol. 104, no. 6, pp. 505-510, 2010.

[59] R. Haque, D. Mondal, P. Duggal et al., "Entamoeba histolytica infection in children and protection from subsequent amebiasis," Infection and Immunity, vol. 74, no. 2, pp. 904-909, 2006.

[60] M. K. Puthia, A. Vaithilingam, J. Lu, and K. S. W. Tan, "Degradation of human secretory immunoglobulin a by Blastocystis," Parasitology Research, vol. 97, no. 5, pp. 386-389, 2005.

[61] M. K. Puthia, J. Lu, and K. S. W. Tan, “Blastocystis ratti contains cysteine proteases that mediate interleukin-8 response from human intestinal epithelial cells in an NF-kappaB-dependent manner," Eukaryotic Cell, vol. 7, no. 3, pp. 435-443, 2008. 

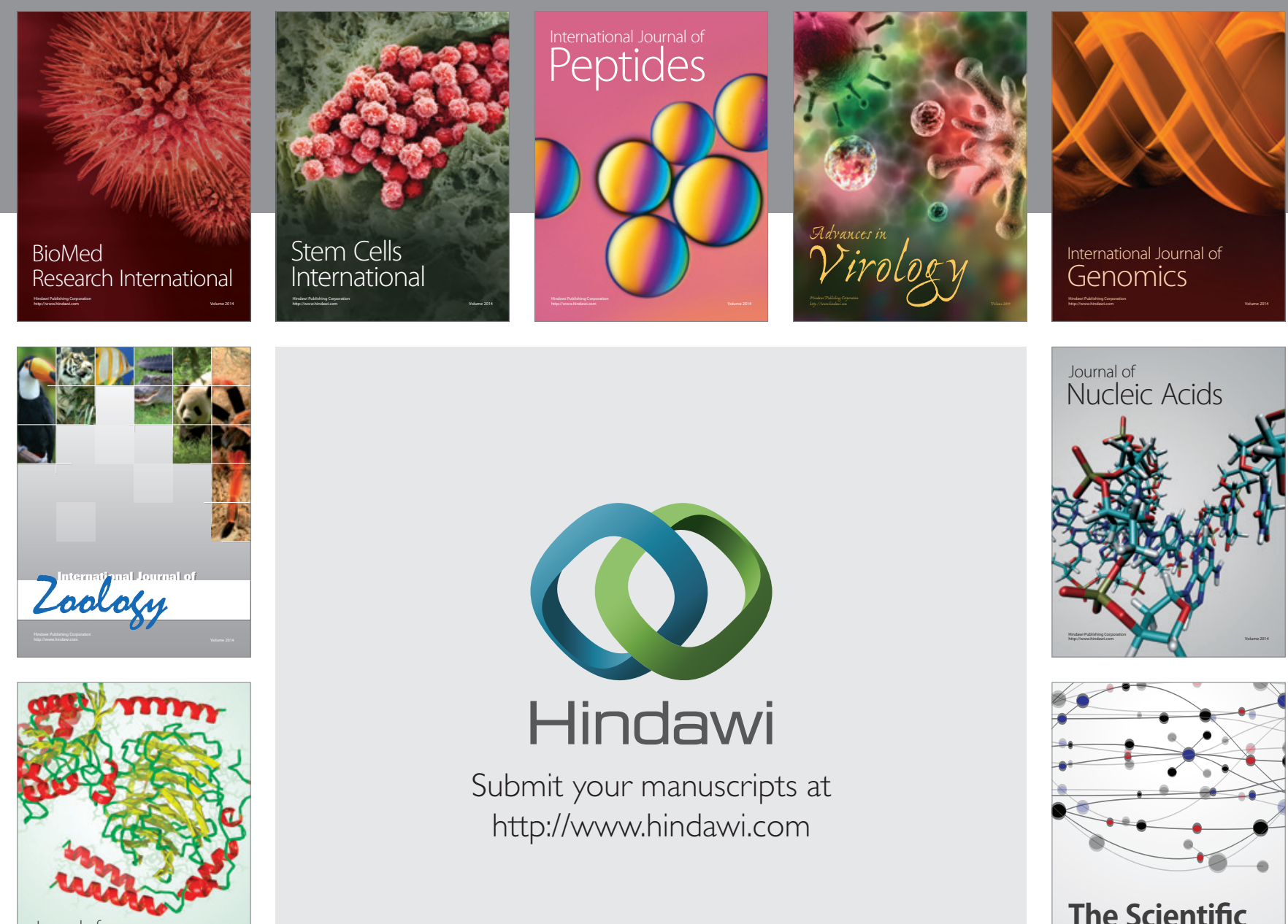

Submit your manuscripts at

http://www.hindawi.com

Journal of
Signal Transduction
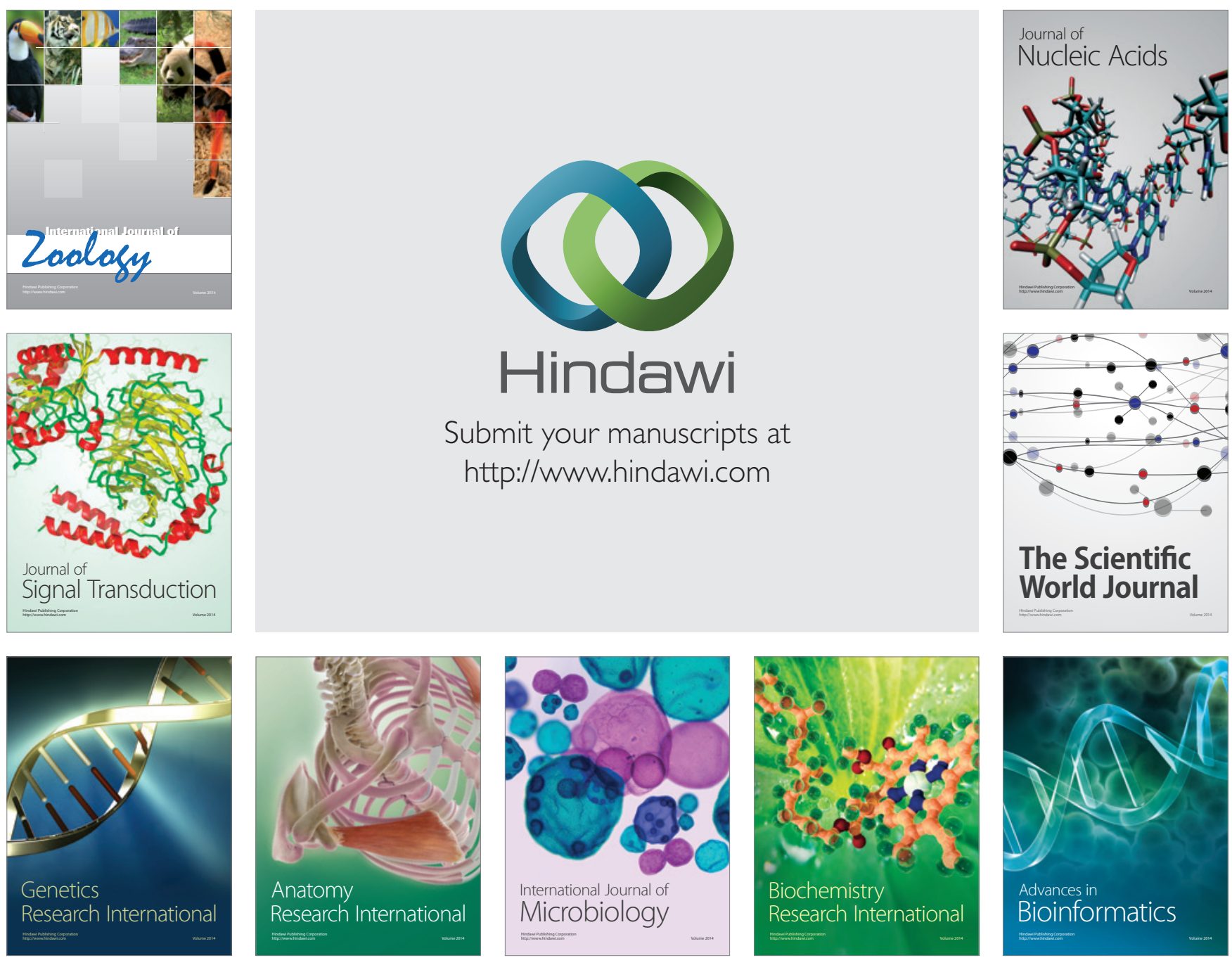

The Scientific World Journal
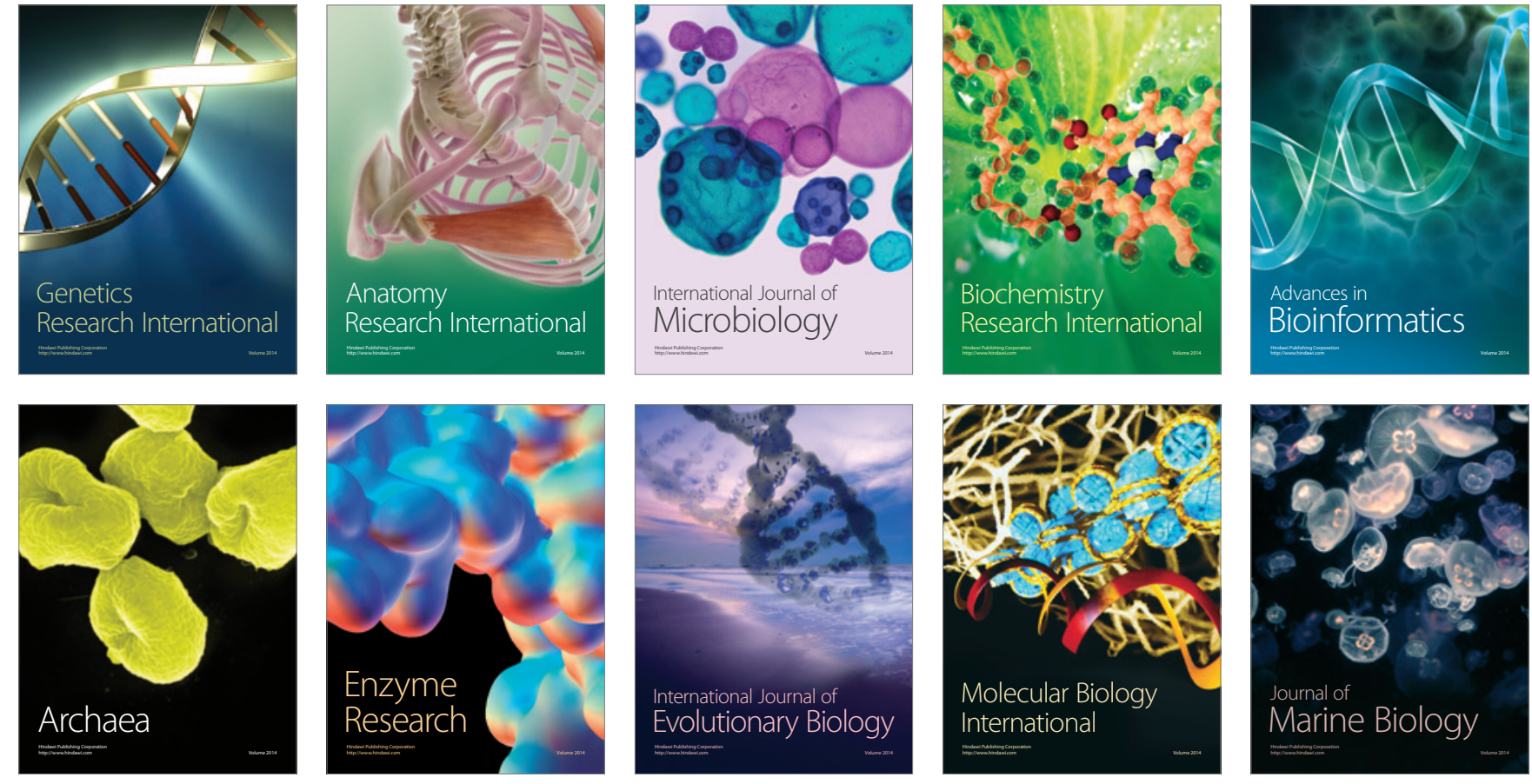